University of Wollongong

Research Online

Faculty of Informatics - Papers (Archive)

Faculty of Engineering and Information

Sciences

$1-1-2009$

\title{
Differential space-time-frequency codes for MB-OFDM UWB with dual carrier modulation
}

Alfred Mertins

University of Luebeck, Germany, mertins@uow.edu.au

Le Chung Tran

University of Wollongong, Ictran@uow.edu.au

Follow this and additional works at: https://ro.uow.edu.au/infopapers

Part of the Physical Sciences and Mathematics Commons

\section{Recommended Citation}

Mertins, Alfred and Tran, Le Chung: Differential space-time-frequency codes for MB-OFDM UWB with dual carrier modulation 2009, 1-5.

https://ro.uow.edu.au/infopapers/787

Research Online is the open access institutional repository for the University of Wollongong. For further information contact the UOW Library: research-pubs@uow.edu.au 


\title{
Differential space-time-frequency codes for MB-OFDM UWB with dual carrier modulation
}

\author{
Abstract \\ In a multiple-input multiple-output (MIMO) multiband orthogonal frequency division multiplexing (MB- \\ OFDM) ultra-wideband (UWB) system, coherent detection where the channel state information (CSI) is \\ assumed to be exactly known at the receiver requires the transmission of a large number of symbols for \\ channel estimation, thus reducing the bandwidth efficiency. This paper examines the use of unitary \\ differential space-time frequency codes (DSTFCs) in MB-OFDM UWB, which increases the system \\ bandwidth efficiency due to the fact that no CSI is required for differential detection. The proposed DSTFC \\ MB-OFDM system would be useful when the transmission of multiple channel estimation symbols is \\ impractical or uneconomical. Simulation results show that the application of DSTFCs associated with \\ dual carrier modulation (DCM) can significantly improve the bit error performance of conventional \\ differential MB-OFDM system (without MIMO), and even provide better bit error performance than the \\ DSTFC MB-OFDM system associated with constant envelope modulation schemes.
}

\section{Keywords}

space, codes, mb, differential, ofdm, frequency, time, uwb, dual, carrier, modulation

\section{Disciplines}

Physical Sciences and Mathematics

\section{Publication Details}

Tran, L. \& Mertins, A. (2009). Differential space-time-frequency codes for MB-OFDM UWB with dual carrier modulation. Proc. IEEE Int. Conf. Communications (ICC 2009) (pp. 1-5). Dresden, Germany: IEEE. 


\title{
Differential Space-Time-Frequency Codes for MB-OFDM UWB with Dual Carrier Modulation
}

\author{
L. C. Tran and A. Mertins
}

University of Luebeck, Germany

\begin{abstract}
In a multiple-input multiple-output (MIMO) multiband orthogonal frequency division multiplexing (MB-OFDM) ultra-wideband (UWB) system, coherent detection where the channel state information (CSI) is assumed to be exactly known at the receiver requires the transmission of a large number of symbols for channel estimation, thus reducing the bandwidth efficiency. This paper examines the use of unitary differential space-time frequency codes (DSTFCs) in MB-OFDM UWB, which increases the system bandwidth efficiency due to the fact that no CSI is required for differential detection. The proposed DSTFC MB-OFDM system would be useful when the transmission of multiple channel estimation symbols is impractical or uneconomical. Simulation results show that the application of DSTFCs associated with dual carrier modulation (DCM) can significantly improve the bit error performance of conventional differential MB-OFDM system (without MIMO), and even provide better bit error performance than the DSTFC MB-OFDM system associated with constant envelope modulation schemes.
\end{abstract}

Index Terms-UWB, MB-OFDM, DSTFC, STFC, MIMO.

\section{INTRODUCTION}

The combination of the emerging technologies, namely multi-band orthogonal frequency division multiplexing ultrawideband (MB-OFDM UWB) [1], multiple-input multipleoutput (MIMO) and space-time-frequency codes (STFCs), to which we will refer as STFC MB-OFDM UWB systems, has been considerably examined in the literature, such as [2], [3], [4], [5], [6], [7], [8]. In all of these works, channel state information (CSI) is assumed to be known exactly at the receiver, thus allowing the receiver to perform coherent detection.

According to [1], six MB-OFDM symbols are transmitted in the physical layer convergence protocol (PLCP) preamble for channel estimation between each pair of transmit (Tx) and receive ( $\mathrm{Rx})$ antennas, thus facilitating coherent detection at the receiver. In a MIMO system consisting of $M \mathrm{Tx}$ and $N \mathrm{Rx}$ antennas, the required number of symbols for this purpose might be as large as $6 M N$, except for the case where superimposed training techniques, such as in [9], [10], [11], are used to reduce the number of channel estimation symbols transmitted within the preamble. Therefore, transmission of a large number of MB-OFDM symbols for channel estimation reduces significantly the system bandwidth efficiency. In fast fading channels or in very high data rate systems, transmission of a large number of MB-OFDM symbols for channel estimation is a hassling task and might even be impractical or uneconomical. In these cases, non-coherent detection (or differential detection), where no CSI is required for decoding signals at the receiver, would be the best candidate.

Although various techniques have been considered in the literature for differential transmission in general OFDM systems associated with a MIMO model, such as [12], [13], [14], [15], [16] and [17], the differential transmission in MB-OFDM systems associated with MIMO has not been thoroughly examined. In this paper, we propose for the first time the framework of differential space-time-frequency codes (DSTFCs) in MB-OFDM UWB communications associated with the dual carrier modulation (DCM) scheme[1], [18]. The DCM scheme, that map a group of four binary bits into two different 16-point constellations, is normally used for the MB-OFDM UWB system at the data rate higher than 200 Mbps [1]. The maximum likelihood (ML) decoding method, that possesses relatively low complexity, is also derived for DSTFCs.

The paper is organized as follows. In Section II, we propose the mathematical model of the proposed DSTFC MB-OFDM UWB system in the case of constant envelope modulation schemes, such as PSK or 4QAM. Section III proposes the DSTFC MB-OFDM UWB system in the case of the DCM scheme, and derives the decoding metrics for the proposed DSTFC. Simulation results are mentioned in Section IV and conclusions are derived in Section V.

Notations: Throughout this paper, the superscripts (.)*, $(.)^{T}$ and $(.)^{H}$ denote the complex conjugation, transposition operation and conjugate transpose operation respectively. We denote $\overline{\mathbf{a}}_{j} \bullet \overline{\mathbf{b}}_{j}$ to be the element-wise (or Hadamard) product between the two vectors $\overline{\mathbf{a}}_{j}$ and $\overline{\mathbf{b}}_{j}$ respectively. Further, $\overline{\mathbf{a}}$. ^2 denotes the element-wise power- 2 operation of $\overline{\mathbf{a}}$. We define the multiplication operation $\mathbf{C} \circ \mathbf{D}$ between the two matrices $\mathbf{C}=\left\{\overline{\mathbf{c}}_{t, m}\right\}_{T \times M}$ and $\mathbf{D}=\left\{\overline{\mathbf{d}}_{m, n}\right\}_{M \times N}$, whose elements $\overline{\mathbf{c}}_{t, m}$ and $\overline{\mathbf{d}}_{m, n}$ are column vectors of the same length, such that the $(t, n)$-th element of the resulting matrix is a column vector $\sum_{m=1}^{M} \overline{\mathbf{c}}_{t, m} \bullet \overline{\mathbf{d}}_{m, n}$. Denote $N_{f f t}$ to be the FFT/IFFT size (for MB-OFDM UWB communications [1], $N_{f f t}=128$ ). Further, $\Re\{c\}$ and $\Im\{c\}$ denote the real and imaginary parts of the complex number $c$. The notation $\operatorname{diag}\left(\overline{\mathbf{a}}_{j}\right)$ denotes a square diagonal matrix formed by stacking the vector $\overline{\mathbf{a}}_{j}$ on the main diagonal of the matrix, while $\left\{\operatorname{diag}\left(\overline{\mathbf{a}}_{j}\right)\right\}_{M_{j} \times N_{j}}$ denotes a $M_{j} \times N_{j}$-sized rectangular matrix whose elements are diagonal matrices $\operatorname{diag}\left(\overline{\mathbf{a}}_{j}\right)$. We state that the two subsets of indices $\{m, k\}$ and $\{m, k\}$ to be different, denoted as $\{m, k\} \neq\{m, k\}$, if at least one of the following two inequalities $m \neq \dot{m}$ and $k \neq k^{\prime}$ occurs. Finally, we define $\overline{\mathbf{1}}$ as a column vector of length $N_{D}$, whose elements are all 1 .

\section{DSTFC MB-OFDM UWB WITH CONSTANT ENVELOPE SigNAL CONSTELLATIONS}

The model of a DSTFC MB-OFDM UWB system in this case is depicted in Fig. 1. As opposed to the STFC MB-OFDM system mentioned above where channels are normally assumed to be constant during multiple blocks of the transmitted STFC, in the DSTFC MB-OFDM system, it is required that channels must be constant during at least two consecutive blocks of the transmitted DSTFC. Thus the DSTFC MB-OFDM system is better fit for fast fading channels. Let us assume generally that channels in the DSTFC MB-OFDM system are constant during a time window of size $2 K T_{S Y M}$ (ns) where $K$ is an integer 


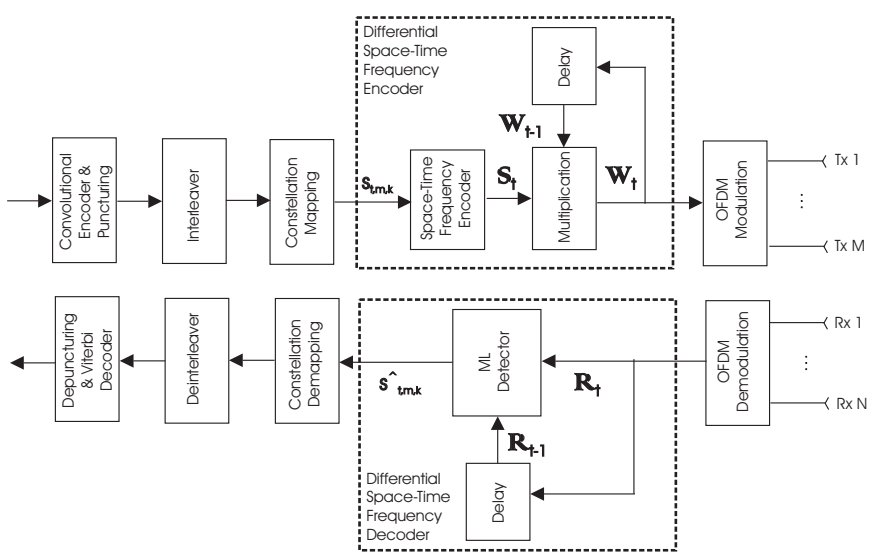

Fig. 1. The proposed DSTFC MB-OFDM UWB system with constant envelope signal constellations.

and $T_{S Y M}$ is the MB-OFDM symbol interval $T_{S Y M}=312.5$ ns [1].

We consider the application of the Alamouti STFC

$$
\mathbf{S}_{\mathbf{t}}=\overline{\mathbf{1}} / \sqrt{2} \circ\left[\begin{array}{rr}
\overline{\mathbf{s}}_{t, 1} & \overline{\mathbf{s}}_{t, 2} \\
-\overline{\mathbf{s}}_{t, 2}^{*} & \overline{\mathbf{s}}_{t, 1}^{*}
\end{array}\right],
$$

where the MB-OFDM symbol $\overline{\mathbf{s}}_{t, m}$, for $m=1,2$, is a column vector of $N_{f f t}$ data corresponding to $N_{f f t}$ sub-carriers, i.e. $\overline{\mathbf{s}}_{t, m}=\left[\begin{array}{ll}s_{t, m, 1} & s_{t, m, 2} \ldots s_{t, m, N_{f f t}}\end{array}\right]^{T}$. Further, we assume that the normalized power of each symbol $s_{t, m, k}$ within $\overline{\mathbf{s}}_{t, m}$, for $k=1, \ldots, N_{f f t}$, is unitary, i.e. $\left|s_{t, m, k}\right|^{2}=1$. Hence $s_{t, m, k}$ can be drawn from a PSK or 4QAM signal constellation, that is denoted as $\mathcal{C}$.

The STFC in (1) can be rewritten in the following form

$$
\mathbb{S}_{t}=1 / \sqrt{2}\left[\begin{array}{rr}
\operatorname{diag}\left(\overline{\mathbf{s}}_{t, 1}\right) & \operatorname{diag}\left(\overline{\mathbf{s}}_{t, 2}\right) \\
-\operatorname{diag}\left(\overline{\mathbf{s}}_{t, 2}^{*}\right) & \operatorname{diag}\left(\overline{\mathbf{s}}_{t, 1}^{*}\right)
\end{array}\right] .
$$

Because the symbols $s_{t, m, k}$ are drawn from a unitary signal constellation, it is easy to realize that $\mathbb{S}_{t}$ is a unitary matrix of size $2 N_{f f t}$, i.e.

$$
\mathbb{S}_{t} \mathbb{S}_{t}^{H}=I_{2 N_{f f t}} .
$$

The proposed DSTFC MB-OFDM system initializes the transmission with an identity matrix $\mathbb{W}_{0}=I_{2 N_{f f t}}$. The following code matrices will be generated and transmitted according to the below principle

$$
\mathbb{W}_{t}=\mathbb{S}_{t} \mathbb{W}_{t-1}
$$

The assumption of channels being constant within a time window $2 K T_{S Y M}$ is equivalent to the fact that channels are constant during the transmission of $K$ consecutive Alamouti code blocks. Therefore, the encoding principle (4) should be applied for $t=1, \ldots,(K-1)$ and the whole transmission protocol is reset for a new time window. Since $\mathbb{S}_{t}$ is a unitary matrix, every transmitted code block $\mathbb{W}_{t}$ is also a unitary one, i.e. $\mathbb{W}_{t} \mathbb{W}_{t}^{H}=I_{2 N_{f f t}}$. The transmission model can be expressed as follows

$$
\mathbb{R}_{\mathbf{t}}=\mathbb{W}_{t} \mathbb{H}_{t}+\mathbb{N}_{t}
$$

The maximum likelihood (ML) decoding metric for the proposed DSTFC is derived as follows. We first represent the STFC in (2) in the following form

$$
\mathbb{S}_{t}=1 / \sqrt{2} \sum_{m=1}^{2} \sum_{k=1}^{N_{f f t}}\left(\mathbb{X}_{t, m, k} s_{t, m, k}^{R}+i \mathbb{Y}_{t, m, k} s_{t, m, k}^{I}\right),
$$

where $s_{t, m, k}^{R}$ and $s_{t, m, k}^{I}$ are the real and imaginary parts of the symbol $s_{t, m, k}$ respectively, i.e. $s_{t, m, k}=s_{t, m, k}^{R}+i s_{t, m, k}^{I}$, while $\mathbb{X}_{t, m, k}$ and $\mathbb{Y}_{t, m, k}$ are their corresponding weighting matrices. We recall that the symbols $s_{t, m, k} \mathrm{~s}$ are drawn from the original PSK or 4QAM signal constellation.

The weighting matrices $\mathbb{X}_{t, m, k}$ and $\mathbb{Y}_{t, m, k}$ in the matrix (2) always satisfy the following properties for a given value $t$

$$
\begin{aligned}
& \mathbb{X}_{t, m, k} \mathbb{X}_{t, m, k}^{H}=\mathbb{Y}_{t, m, k} \mathbb{Y}_{t, m, k}^{H} \quad \forall m, k, \\
& \mathbb{X}_{t, m, k} \mathbb{X}_{t, \dot{m}, \dot{k}}^{H}=-\mathbb{X}_{t, \dot{m}, \dot{k}} \mathbb{X}_{t, m, k}^{H}, \forall\{m, k\} \neq\{\dot{m}, \hat{k}\}, \\
& \mathbb{Y}_{t, m, k} \mathbb{Y}_{t, \dot{m}, \dot{k}}^{H}=-\mathbb{Y}_{t, \dot{m}, \dot{k}} \mathbb{Y}_{t, m, k}^{H}, \forall\{m, k\} \neq\{\dot{m}, \dot{k}\}, \\
& \mathbb{X}_{t, m, k} \mathbb{Y}_{t, \dot{m}, \dot{k}}^{H}=\mathbb{Y}_{t, \dot{m}, \dot{k}} \mathbb{X}_{t, m, k}^{H} \quad \forall m, k, \dot{m}, \dot{k} .
\end{aligned}
$$

To formulate the ML decoding metric for the symbol $s_{t, m, k}$, for $t=1, \ldots,(K-1), m=1,2$ and $k=1, \ldots, N_{f f t}$, let us consider the following term

$$
D_{m, k}=D_{m, k}^{R}+i D_{m, k}^{I},
$$

where $D_{m, k}^{R}=\Re\left[\operatorname{tr}\left(\mathbb{R}_{t-1} \mathbb{R}_{t}^{H} \mathbb{X}_{t, m, k}\right)\right]$ and $D_{m, k}^{I}=$ $\Re\left[\operatorname{tr}\left(\mathbb{R}_{t-1} \mathbb{R}_{t}^{H} i \mathbb{Y}_{t, m, k}\right)\right]$. We have

$$
\begin{aligned}
D_{m, k}^{R} & =\Re\left[\operatorname{tr}\left(\mathbb{R}_{t-1} \mathbb{R}_{t}^{H} \mathbb{X}_{t, m, k}\right)\right] \\
& =\Re\left\{\operatorname{tr}\left[\left(\mathbb{W}_{t-1} \mathbb{H}_{t-1}+\mathbb{N}_{t-1}\right)\left(\mathbb{W}_{t} \mathbb{H}_{t}+\mathbb{N}_{t}\right)^{H} \mathbb{X}_{t, m, k}\right]\right\} \\
& =\Re\left\{\operatorname{tr}\left[\left(\left(\mathbb{W}_{t-1} \mathbb{H}_{t-1} \mathbb{H}_{t}^{H} \mathbb{W}_{t-1}^{H} \mathbb{S}_{t}^{H}\right)+\mathbb{N}\right) \mathbb{X}_{t, m, k}\right]\right\},
\end{aligned}
$$

where $\mathbb{N}:=\mathbb{W}_{t-1} \mathbb{H}_{t-1} \mathbb{N}_{t}^{H}+\mathbb{N}_{t-1} \mathbb{H}_{t}^{H} \mathbb{W}_{t}^{H}+\mathbb{N}_{t-1} \mathbb{N}_{t}^{H}$. Because $\mathbb{W}_{t-1}$ is a unitary matrix, i.e.

$$
\mathbb{W}_{t-1} \mathbb{W}_{t-1}^{H}=I_{2 N_{f f t}},
$$

and channel coefficients are constant during a time window of $K$ code blocks, i.e. $\mathbb{H}_{t}=\mathbb{H}_{t-1}$, Eq. (11) becomes

$$
D_{m, k}^{R}=\Re\left\{\operatorname{tr}\left[\left(\mathbb{H}_{t} \mathbb{H}_{t}^{H}\right)\left(\mathbb{S}_{t}^{H} \mathbb{X}_{t, m, k}\right)\right]\right\}+\Re\left\{\operatorname{tr}\left(\mathbb{N}_{t, m, k}\right)\right\} .
$$

The first term is calculated as follows

$$
\begin{aligned}
\Re\left\{\operatorname{tr}\left(\mathbb{H}_{t} \mathbb{H}_{t}^{H} \mathbb{S}_{t}^{H} \mathbb{X}_{t, m, k}\right)\right\}= \\
1 / \sqrt{2} \Re\left\{\operatorname { t r } \left[\mathbb { H } _ { t } \mathbb { H } _ { t } ^ { H } \left(\mathbb{X}_{t, m, k}^{H} \mathbb{X}_{t, m, k} s_{t, m, k}^{R}\right.\right.\right. \\
+\sum_{\forall \dot{m}, \dot{k},\{\dot{m}, \dot{k}\} \neq\{m, k\}} \mathbb{X}_{t, \dot{m}, \dot{k}}^{H} \mathbb{X}_{t, m, k} s_{t, \dot{m}, \dot{k}}^{R} \\
\left.\left.\left.+i \sum_{\forall \dot{m}, \dot{k}} \mathbb{Y}_{t, \dot{m}, \dot{k}}^{H} \mathbb{X}_{t, m, k} s_{t, \dot{m}, \dot{k}}^{I}\right)\right]\right\} .
\end{aligned}
$$

It is noted that if $\Phi$ is an antihermitian (or skew-Hermitian) matrix, i.e. $\Phi^{H}=-\Phi$, then $\operatorname{tr}\left(\mathbb{A}^{H} \mathbb{A} \Phi\right)$ is imaginary, thus $\Re\left\{\operatorname{tr}\left(\mathbb{A}^{H} \mathbb{A} \Phi\right)\right\}=0$. From (7), it is easy to check that $\left(\mathbb{X}_{t, \dot{m}, \dot{k}}^{H} \mathbb{X}_{t, m, k} s_{t, \dot{m}, \dot{k}}^{R}\right)^{H}=-\left(\mathbb{X}_{t, \dot{m}, \dot{k}}^{H} \mathbb{X}_{t, m, k} s_{t, \dot{m}, \dot{k}}^{R}\right)$, i.e. $\left(\mathbb{X}_{t, \dot{m}, k}^{H} \mathbb{X}_{t, m, k} s_{t, m, k}^{R}\right)$ is an antihermitian matrix, thus

$$
\left.\Re\left\{\operatorname{tr}\left(\mathbb{H}_{t} \mathbb{H}_{t}^{H} \mathbb{X}_{t, \dot{m}, \dot{k}}^{H} \mathbb{X}_{t, m, k} s_{t, \dot{m}, \dot{k}}^{R}\right)\right)\right\}=0,
$$

for all $\dot{m}, \dot{k}$ and $\{\dot{m}, \dot{k}\} \neq\{m, k\}$.

On the other hand, if $\Theta$ is a Hermitian matrix, i.e. $\Theta^{H}=\Theta$, then $\operatorname{tr}\left(\mathbb{A}^{H} \mathbb{A} \Theta\right)$ is real, thus $\Im\left\{\operatorname{tr}\left(\mathbb{A}^{H} \mathbb{A} \Theta\right)\right\}=0$. 
From (9), it is trivial to realize that $\left(\mathbb{Y}_{t, \dot{m}, k}^{H} \mathbb{X}_{t, m, k} s_{t, k}^{I}\right)^{H}=$ $\left(\mathbb{Y}_{t, \dot{m}, \hat{k}}^{H} \mathbb{X}_{t, m, k} s_{t, k}^{I}\right)$, i.e. $\left(\mathbb{Y}_{t, \dot{m}, \hat{k}}^{H} \mathbb{X}_{t, m, k} s_{t, k}^{I}\right)$ is a Hermitian matrix, thus

$$
\begin{aligned}
\Re\left\{\operatorname{tr}\left(\mathbb{H}_{t} \mathbb{H}_{t}^{H} \mathbb{Y}_{t, \dot{m}, \dot{k}}^{H} i \mathbb{X}_{t, m, k} s_{t, \dot{m}, \dot{k}}^{I}\right)\right\} \\
=\Im\left\{\operatorname{tr}\left(\mathbb{H}_{t} \mathbb{H}_{t}^{H} \mathbb{Y}_{t, \dot{m}, \dot{k}}^{H} \mathbb{X}_{t, m, k} s_{t, \dot{m}, \dot{k}}^{I}\right)\right\} \\
=0 \quad \forall m, k, \dot{m}, \dot{k} .
\end{aligned}
$$

If we denote $\mathbb{C}_{t, m, k}:=\mathbb{H}_{t} \mathbb{H}_{t}^{H} \mathbb{X}_{t, m, k}^{H} \mathbb{X}_{t, m, k}$ then $\mathbb{C}_{t, m, k}$ is a constant matrix for given values $t, m$ and $k$ and $\operatorname{tr}\left(\mathbb{C}_{t, m, k}\right)$ is a positive real number (the trivial case $\operatorname{tr}\left(\mathbb{C}_{t, m, k}\right)=0$ is discarded). From (12)-(15), we have

$$
D_{m, k}^{R}=1 / \sqrt{2} \operatorname{tr}\left(\mathbb{C}_{t, m, k}\right) s_{t, m, k}^{R}+\Re\left\{\operatorname{tr}\left(\mathbb{N}_{t, m, k}\right)\right\} .
$$

The term $D_{m, k}^{I}$ is calculated in a similar way with the note that $\mathbb{H}_{t} \mathbb{H}_{t}^{H} \mathbb{Y}_{t, m, k} \mathbb{Y}_{t, m, k}^{H}=\mathbb{H}_{t} \mathbb{H}_{t}^{H} \mathbb{X}_{t, m, k} \mathbb{X}_{t, m, k}^{H}=\mathbb{C}_{t, m, k}$ (cf. Eq. (6)), we have

$$
D_{m, k}^{I}=1 / \sqrt{2} \operatorname{tr}\left(\mathbb{C}_{t, m, k}\right) s_{t, m, k}^{I}+\Re\left\{\operatorname{tr}\left(\mathbb{N} i \mathbb{Y}_{t, m, k}\right)\right\} .
$$

Therefore

$$
\begin{aligned}
D_{m, k}= & D_{m, k}^{R}+i D_{m, k}^{I} \\
= & 1 / \sqrt{2} \operatorname{tr}\left(\mathbb{C}_{t, m, k}\right) s_{t, m, k}+ \\
& \Re\left\{\operatorname{tr}\left(\mathbb{N}_{t, m, k}\right)\right\}+i \Re\left\{\operatorname{tr}\left(\mathbb{N} i \mathbb{Y}_{t, m, k}\right)\right\} .
\end{aligned}
$$

The ML decoding metric for $s_{t, m, k}$ can be derived as follows

$$
\begin{aligned}
\hat{s}_{t, m, k}= & \arg \min \left|D_{m, k}-\frac{1}{\sqrt{2}} \operatorname{tr}\left(\mathbb{C}_{t, m, k}\right) s_{t, m, k}\right|^{2} \\
= & \arg \min \left(\left|D_{m, k}\right|^{2}+\frac{1}{2}\left[\operatorname{tr}\left(\mathbb{C}_{t, m, k}\right)\right]^{2}\left|s_{t, m, k}\right|^{2}\right. \\
& \left.-\sqrt{2} \operatorname{tr}\left(\mathbb{C}_{t, m, k}\right) \Re\left\{D_{m, k}^{*} s_{t, m, k}\right\}\right) \\
= & \arg \min \left(\left|D_{m, k}\right|^{2}+\frac{1}{2}\left[\operatorname{tr}\left(\mathbb{C}_{t, m, k}\right)\right]^{2}\right. \\
& \left.-\sqrt{2} \operatorname{tr}\left(\mathbb{C}_{t, m, k}\right) \Re\left\{D_{m, k}^{*} s_{t, m, k}\right\}\right) .
\end{aligned}
$$

Since $\mathbb{C}_{t, m, k}$ is a constant matrix for given $t, m$ and $k$ and $\operatorname{tr}\left(\mathbb{C}_{t, m, k}\right)$ is a positive real number, the equivalent ML decoding metric for $s_{t, m, k}$ is

$$
\hat{s}_{t, m, k}=\arg \max _{s_{t, m, k} \in \mathcal{C}}\left(\Re\left\{D_{m, k}^{*} s_{t, m, k}\right\}\right),
$$

for $t=1, \ldots,(K-1), m=1,2$ and $k=1, \ldots, N_{f f t}$. In fact, there are only $N_{D}=100$ data symbols within each MBOFDM symbol, that includes 28 other pilot, guard and null symbols [1]. Therefore, instead of decoding $N_{f f t}$ symbols in Eq. (16), i.e. $k=1, \ldots, N_{f f t}$, we only need to decode $N_{D}$ symbols, i.e. $k=1, \ldots, N_{D}$.

Eq. (16) means that each of the two MB-OFDM symbols $\overline{\mathbf{s}}_{\mathbf{t}, \mathbf{1}}$ and $\overline{\mathbf{s}}_{\mathbf{t}, \mathbf{2}}$ can be separately decoded. Furthermore, each symbol $s_{t, m, k}$ within these two MB-OFDM symbols can also be separately decoded based on the above equation. In other words, instead of jointly decoding all $2 N_{f f t}$ symbols $s_{t, m, k}$ within the two MB-OFDM symbols $\overline{\mathbf{s}}_{\mathbf{t}, \mathbf{1}}$ and $\overline{\mathbf{s}}_{\mathbf{t}, \mathbf{2}}$ at a time, each of them can be separately decoded. No CSI is required for the decoding process. All we need for the decoding process at time $t$ are the received signals at the previous time $(t-1)$ and at the current time. The decoding process is completely linear, thus relatively simple.
TABLE I

DCM MAPPING TABLE [1]

\begin{tabular}{l|r|r||l|r|r}
\hline Input bits & $d[n]$ & $d[n+50]$ & Input bits & $d[n]$ & $d[n+50]$ \\
\hline 0000 & $-3-3 i$ & $1+i$ & 1000 & $1-3 i$ & $3+i$ \\
0001 & $-3-1 i$ & $1-3 i$ & 1001 & $1-i$ & $3-3 i$ \\
0010 & $-3+i$ & $1+3 i$ & 1010 & $1+i$ & $3+3 i$ \\
0011 & $-3+3 i$ & $1-i$ & 1011 & $1+3 i$ & $3-i$ \\
0100 & $-1-3 i$ & $-3+i$ & 1100 & $3-3 i$ & $-1+i$ \\
0101 & $-1-i$ & $-3-3 i$ & 1101 & $3-i$ & $-1-3 i$ \\
0110 & $-1+i$ & $-3+3 i$ & 1110 & $3+i$ & $-1+3 i$ \\
0111 & $-1+3 i$ & $-3-i$ & 1111 & $3+3 i$ & $-1-i$ \\
\hline
\end{tabular}

\section{DSTFC MB-OFDM UWB WITH DCM}

\section{A. DCM}

For the data rates being higher than $200 \mathrm{Mpbs}$, DCM, which is a multi-dimensional constellation, will be used instead of QPSK to employ better the frequency and time diversities, thus providing better error performance over QPSK. The coded and interleaved binary serial input data shall be divided into groups of 200 bits and converted into 100 complex numbers. The conversion shall be performed as follows

1) The 200 coded and interleaved bits are grouped into 50 groups of 4 bits. Each group is represented as $(b[g(n)], b[g(n)+1], b[g(n)+50)], b[g(n)+51])$, where $g(n)=2 n$ if $n \in[0,24]$, and $g(n)=2 n+50$ if $n \in[25,49]$.

2) Each group of four bits $(b[g(n)], b[g(n)+1], b[g(n)+$ $50)], b[g(n)+51])$ shall be mapped into two different 16-point constellations, i.e. two complex numbers $(d[n], d[n+50])$, separated by 50 tones (sub-carriers). The mapping between bits and constellation is enumerated in Table I.

3) The complex numbers shall be normalized using a normalization factor $1 / \sqrt{10}$ to have a unitary normalized average power.

Therefore, after DCM modulation and before OFDM modulation, $N_{D}=100$ data symbols are allocated in such a way that the first $N_{D} / 2=50$ symbols $s_{t, m, k}$, for $k=1, \ldots, 50$, are taken from the pool of symbols listed in the 2nd and 5th columns in Table I after being normalized, which is denoted as $\mathcal{C}_{\mathcal{D C M}}$, while the last 50 symbols $s_{t, m, k+50}$ are taken from the pool of symbols listed in the 3rd and 6th columns after being normalized, which is denoted as $\mathcal{C}_{\mathcal{D C}} \mathcal{M}_{50}$. It is easy to realize that $\left|s_{t, m, k}\right|^{2}+\left|s_{t, m, k+50}\right|^{2}=2$ for $\forall k, k=1, \ldots, 50$.

\section{B. The Proposed System and ML Decoding Method}

The proposed DSTFC MB-OFDM system in the case of DCM is depicted in Fig. 2. Comparing it with Fig. 1, one can realize that the two new block, namely multiplexer (MUX) and demultiplexer (DEMUX), are added.

At the transmitter, the MUX block swaps the position of the last 50 data symbols $s_{t, 1, k+50}$, for $k=1, \ldots, 50$, within the MB-OFDM symbol $\overline{\mathbf{s}}_{t, 1}$ and that of the first 50 symbols $s_{t, 2, k}, k=1, \ldots, 50$, within the MB-OFDM symbol $\overline{\mathbf{s}}_{t, 2}$. By doing this, two new MB-OFDM symbols, denoted as $\overline{\mathbf{s}}_{t, 1}$ and $\overline{\mathbf{s}}_{t, 2}$, are generated. Thus we can present $\overline{\mathbf{s}}_{t, 1}$ and $\overline{\mathbf{s}}_{t, 2}$ as

$$
\begin{aligned}
& \overline{\mathbf{s}}_{t, 1}=\left[s_{t, 1,1}, \ldots, s_{t, 1,50}, s_{t, 2,1}, \ldots, s_{t, 2,50}\right]^{T} \\
& \overline{\mathbf{s}}_{t, 2}=\left[s_{t, 1,51}, \ldots, s_{t, 1,100}, s_{t, 2,51}, \ldots, s_{t, 2,100}\right]^{T} .
\end{aligned}
$$

We denote $\overline{\mathbf{s}}_{t, 1}=\left[\dot{s}_{t, 1,1}, \ldots, \dot{s}_{t, 1,100}\right]^{T}$ and $\overline{\mathbf{s}}_{t, 2}=$ $\left[\hat{s}_{t, 2,1}, \ldots, \hat{s}_{t, 2,100}\right]^{T}$. It is noted that all symbols within $\overline{\mathbf{s}}_{t, 1}$ belong to the symbol pool $\mathcal{C}_{\mathcal{D C M}}$, while all symbols within $\overline{\mathbf{s}}_{t, 2}$ belong to the symbol pool $\mathcal{C}_{\mathcal{D C M}} \mathcal{M}_{5}$. 


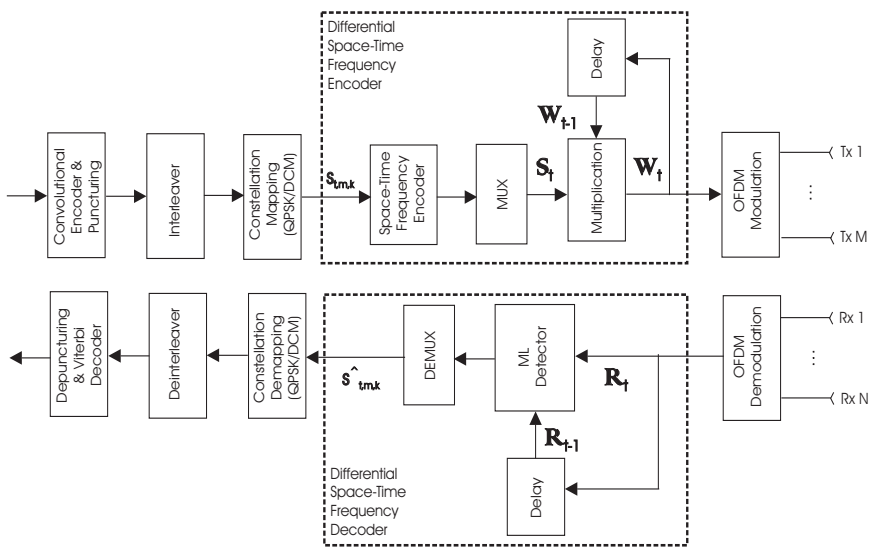

Fig. 2. Structural diagram of the proposed DSTFC MB-OFDM UWB system with DCM.

Since $\left|s_{t, m, k}\right|^{2}+\left|s_{t, m, k+50}\right|^{2}=2$ for $\forall k, k=1, \ldots, 50$, one can realize that

$$
\overline{\mathbf{1}} / \sqrt{2} \circ\left(\left|\overline{\mathbf{s}}_{t, 1}\right| \cdot{ }^{\wedge} 2+\left|\overline{\mathbf{s}}_{t, 2}\right| \cdot{ }^{\wedge} 2\right)=\overline{\mathbf{1}} \text {. }
$$

We use the notations $\mathbb{X}_{t, m, k}, \mathbb{Y}_{t, m, k}$ and $\dot{C}_{t, m, k}$ to denote the matrices that are similar to $\mathbb{X}_{t, m, k}, \mathbb{Y}_{t, m, k}$ and $\mathbb{C}_{t, m, k}$ in Section II, but the role of $s_{t, m, k}$ has been replaced by $\dot{s}_{t, m, k}$. By the proposed structure, the following three conditions have always been assured

1) The normalized transmitted matrix $\mathbb{S}_{t}$ in the DCM case

$$
\mathbb{S}_{t}=1 / \sqrt{2}\left[\begin{array}{rr}
\operatorname{diag}\left(\overline{\mathbf{s}}_{t, 1}\right) & \operatorname{diag}\left(\overline{\mathbf{\mathbf { s }}}_{t, 2}\right) \\
-\operatorname{diag}\left(\overline{\mathbf{s}}_{t, 2}^{*}\right) & \operatorname{diag}\left(\overline{\mathbf{s}}_{t, 1}^{*}\right)
\end{array}\right]
$$

satisfies $\mathbb{S}_{t} \mathbb{S}_{t}^{H}=I_{2 N_{f f t}}$, similarly to (3).

2) The equality $\dot{\mathbb{C}}_{t, 1, k}=\dot{\mathbb{C}}_{t, 2, k}$, i.e. $\dot{\mathbb{X}}_{t, 1, k}^{H} \mathbb{X}_{t, 1, k}=$ $\dot{\mathbb{X}}_{t, 2, k}^{H} \dot{\mathbb{X}}_{t, 2, k}=\mathbb{Y}_{t, 1, k}^{H} \mathbb{Y}_{t, 1, k}=\mathbb{Y}_{t, 2, k}^{H} \mathbb{Y}_{t, 2, k}$, is guaranteed for $\forall t, k$. We denote $\dot{C}_{t, k}:=\stackrel{\mathbb{C}}{t, 1, k}_{t, 2, k}$.

3) We always have $\frac{1}{2}\left(\left|s_{t, 1, k}\right|^{2}+\left|\dot{s}_{t, 2, k}\right|^{2}\right)=1 \forall k, k=$ $1, \ldots, 100$.

As a result, each pair of symbols $\left(s_{t, 1, k}, s_{t, 2, k}\right)$, for $k=$ $1, \ldots, 100$, within the pair of MB-OFDM symbols $\left(\overline{\mathbf{s}}_{t, 1}, \overline{\dot{\mathbf{s}}}_{t, 2}\right)$ can be decoded separately based on the following ML decoding metric, which is resulted from a slight modification of the ML decoding metric in Section II

$$
\begin{aligned}
& \left(\hat{s}_{t, 1, k}, \hat{s}_{t, 2, k}\right) \\
= & \arg \min \sum_{m=1}^{2}\left|D_{m, k}-\frac{1}{\sqrt{2}} \operatorname{tr}\left(\dot{\mathbb{C}}_{t, m, k}\right) \dot{s}_{t, m, k}\right|^{2} \\
= & \arg \min \left[\sum_{m=1}^{2}\left|D_{m, k}\right|^{2}+\frac{1}{2}\left[\operatorname{tr}\left(\dot{\mathbb{C}}_{t, k}\right)\right]^{2} \sum_{m=1}^{2}\left|\dot{s}_{t, m, k}\right|^{2}\right. \\
& \left.-\sqrt{2} \operatorname{tr}\left(\dot{\mathbb{C}}_{t, k}\right) \sum_{m=1}^{2} \Re\left\{D_{m, k}^{*} \dot{s}_{t, m, k}\right\}\right] \\
= & \arg \min \left[\sum_{m=1}^{2}\left|D_{m, k}\right|^{2}+\left[\operatorname{tr}\left(\dot{\mathbb{C}}_{t, k}\right)\right]^{2}\right. \\
& \left.-\sqrt{2} \operatorname{tr}\left(\dot{\mathbb{C}}_{t, k}\right) \sum_{m=1}^{2} \Re\left\{D_{m, k}^{*} \dot{s}_{t, m, k}\right\}\right] .
\end{aligned}
$$

TABLE II SIMULATION PARAMETERS.

\begin{tabular}{l|r}
\hline Parameter & Value \\
\hline FFT and IFFT size & $N_{f f t}=128$ \\
Data rate & $320 \mathrm{Mbps}$ \\
Convolutional encoder's rate & $1 / 2$ \\
Convolutional encoder's constraint length & 7 \\
Convolutional decoder & Viterbi \\
Decoding mode & Hard \\
STFC decoding at nodes & ML decoding \\
Number of transmitted & 1200 \\
DSTFC blocks & QPSK \& DCM \\
Modulation & CM1, $2,3 \& 4$ \\
IEEE Channel model & $N_{D}=100$ \\
Number of data subcarriers & $N_{P}=12$ \\
Number of pilot subcarriers & $N_{G}=10$ \\
Number of guard subcarriers & $N_{T}=122$ \\
Total number of subcarriers used & $N_{Z P S}=37$ \\
Number of samples in ZPS & $N_{S Y}=165$ \\
Total number of samples/symbol & 100 \\
Number of channel realizations & \\
\hline
\end{tabular}

In these formulas, $D_{m, k}$ is calculated in the similar manner as (10) with the role of $s_{t, m, k}$ substituted by $\dot{s}_{t, m, k}$. The above metric is equivalent to the following one

$$
\begin{aligned}
\left(\hat{s}_{t, 1, k}, \hat{s}_{t, 2, k}\right)= & \arg \max _{\hat{s}_{t, 1, k} \in \mathcal{C}_{\mathcal{D C M}}, \hat{s}_{t, 2, k} \in \mathcal{C}_{\mathcal{D C M}} 50} \\
& \Re\left\{D_{1, k}^{*} \dot{s}_{t, 1, k}+D_{2, k}^{*} \dot{s}_{t, 2, k}\right\},
\end{aligned}
$$

for $\forall k, k=1, \ldots, 100$.

After all data symbols within the two MB-OFDM symbols $\overline{\mathbf{s}}_{t, 1}$ and $\overline{\mathbf{s}}_{t, 2}$ are decoded, the DEMUX block swaps back the position of the last 50 data symbols in $\overline{\mathbf{s}}_{t, 1}$ and that of the first 50 data symbols in $\overline{\mathbf{s}}_{t, 2}$. Thereby, the MB-OFDM symbols $\overline{\mathbf{s}}_{t, 1}$ and $\overline{\mathbf{s}}_{t, 2}$ have been completely decoded.

Comparing (16) and (18), one can realize that decoding DSTFCs in the DCM case is slightly more complicated than that in the PSK or 4QAM case, though decoding complexity in both cases is relatively simple.

\section{Simulation Results}

We have run several Monte-Carlo simulations for the baseband, conventional differential MB-OFDM system (without MIMO) with QPSK modulation scheme and the two baseband Alamouti DSTFC MB-OFDM systems with QPSK and DCM modulation schemes. All systems are considered at the bit rate $320 \mathrm{Mbps}$ and with the same total transmission power. Each run of simulations was carried out with 1200 Alamouti DSTFC blocks. One hundred channel realizations of each channel model (CM 1 to CM 4) were considered for the transmission of each DSTFC block. The channel realizations were created by the Matlab program enclosed in the IEEE 802.15.3a channel modeling sub-committee report [19]. In simulations, $S N R$ is defined to be the signal-to-noise ratio $(\mathrm{dB})$ per sample in a MB-OFDM symbol (consisting of 165 samples), at each Rx antenna (i.e. the subtraction between the total power $(\mathrm{dB})$ of the received signal corresponding to the sample of interest and the power of noise $(\mathrm{dB})$ at that Rx antenna). The complete set of simulation parameters is presented in Table II.

Fig. 3 compares the three systems in the case of one $\mathrm{Rx}$ antenna. The proposed DSTFC system with DCM brings about a significant improvement in the bit error performance, compared to the other two systems. For instance, the proposed DCM scheme provides the Alamouti DSTFC MB-OFDM system with an approximate $6 \mathrm{~dB}$ gain in the case of $\mathrm{CM}$ 1 , at the bit error rate $B E R=10^{-4}$, over the QPSK scheme. The more dispersive the channel is, the higher gain the DCM scheme provides. 


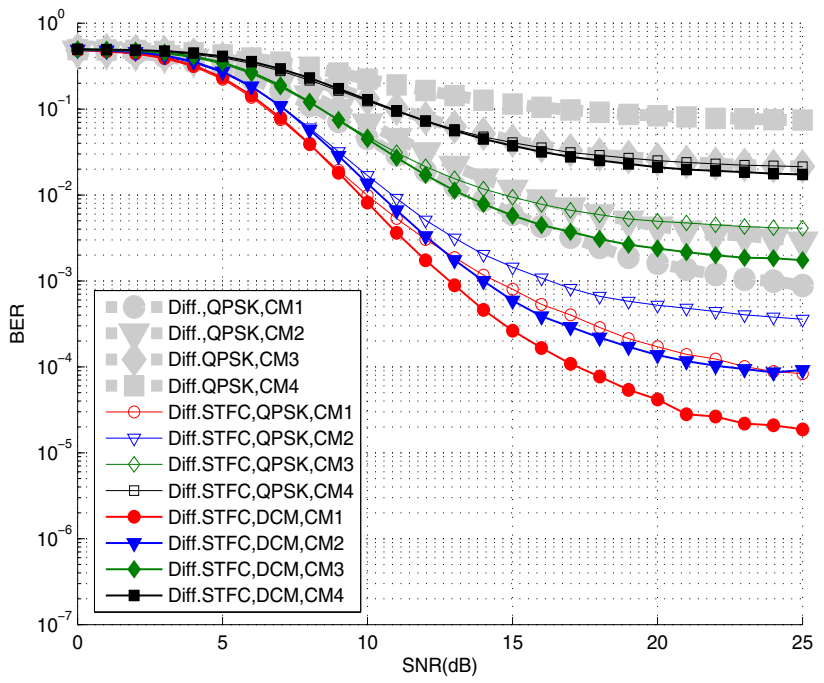

Fig. 3. DSTFC MB-OFDM with DCM and QPSK schemes and with one Rx antenna.

Fig. 4 shows the case of two Rx antennas. Again, we can realize that the proposed DCM scheme improves significantly the bit error performance of DSTFC MB-OFDM systems. For illustration, a gain of at least $3 \mathrm{~dB}$ over the QPSK scheme can be achieved in the Alamouti DSTFC MB-OFDM system at $B E R=10^{-5}$. It is noted that the aforementioned improvements were gained without any increase of total transmission power.

\section{Conclusions}

The paper has proposed the framework of DSTFC MBOFDM UWB systems using the DCM scheme. It has been shown that DSTFC MB-OFDM systems using DCM can possess much better bit error performance, compared to the conventional differential MB-OFDM UWB with PSK or 4QAM schemes, and are significantly better over the DSTFC MBOFDM systems using PSK or 4QAM schemes, with the same total transmission power. Similarly to DSTFC MB-OFDM systems with constant envelope modulation schemes, DSTFC MB-OFDM systems with DCM are also able to be used in various other wireless applications, such as WiMax MIMO [20]. This topic will be addressed in our future works.

\section{ACKNOWLEDGMENT}

L. C. Tran would like to thank the Alexander von Humboldt $(\mathrm{AvH})$ Foundation, Germany, for its support of this work under the form of a postdoctoral fellowship.

\section{REFERENCES}

[1] A. Batra et al., "Multiband OFDM physical layer specification.," WiMedia Alliance Release 1.1, July 2005.

[2] T.-H. Tan and K.-C. Lin, "Performance of space-time block coded MBOFDM UWB systems," Proc. 4th Annual Communication Networks and Services Research Conference (CNSR'06), pp. 323 - 327, May 2006.

[3] W. P. Siriwongpairat, W. Su, M. Olfat, and K. J. R. Liu, "MultibandOFDM MIMO coding framework for UWB communication systems," IEEE Trans. Sign. Process., vol. 54, no. 1, pp. 214 - 224, Jan. 2006.

[4] L. C. Tran and A. Mertins, "Space-time frequency code implementation in MB-OFDM UWB communications: design criteria and performance," accepted for publication in IEEE Trans. Wireless Commun. Available at http://www.isip.uni-luebeck.de/index.php?id=278, 2007.

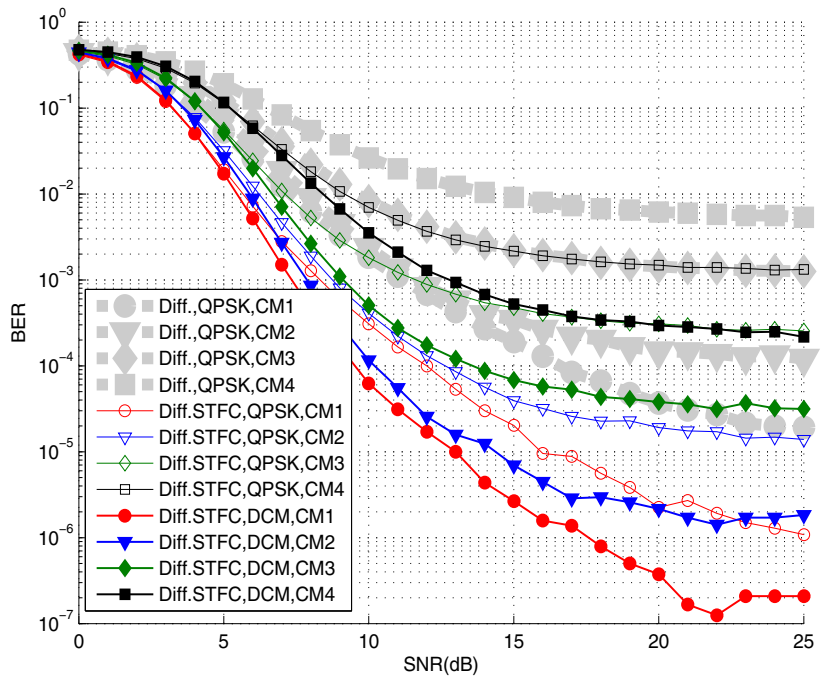

Fig. 4. DSTFC MB-OFDM with DCM and QPSK schemes and with two Rx antennas.

[5] L. C. Tran, A. Mertins, E. Dutkiewicz, and X. Huang, "Space-timefrequency codes in MB-OFDM UWB communications: Advanced order8 STFC and its performance," Proc. 7th IEEE International Symposium on Communications and Information Technologies ISCIT 2007, Oct. 2007.

[6] L. C. Tran, A. Mertins, and T. A. Wysocki, "Quasi-orthogonal spacetime-frequency codes in MB-OFDM UWB communications," Proc. International Conference on Signal Processing and Communication Systems ICSPCS2007, Dec. 2007.

[7] L. C. Tran and A. Mertins, "On the use of quasi-orthogonal space-timefrequency codes in MB-OFDM UWB," Proc. 2nd IEEE International Conference on Communication and Electronics (ICCE2008), pp. 252257, June 2008

[8] L. C. Tran and A. Mertins, "Application of quasi-orthogonal spacetime-frequency codes in MB-OFDM UWB," Proc. IEEE International Conference on Ultra-Wideband (ICUWB 2008)., Sept. 2008.

[9] Q. Yang and K. S. Kwak, "Superimposed training for estimating of doubly-selective OFDM channels," Proc. 9th Int. Conf. Advanced Communication Technology (ICACT 2007), vol. 3, pp. 1652-1655, Feb. 2007.

[10] K. Josiam and D. Rajan, "Bandwidth efficient channel estimation using super-imposed pilots in OFDM systems," IEEE Trans. Wireless Commun., vol. 6, pp. 2234-2245, June 2007.

[11] S. Lu, G. Kang, Q. Zhu, and P. Zhang, "A orthogonal superimposed pilot for channel estimation in MIMO-OFDM systems," Proc. IEEE 65th Vehicular Technology Conference (VTC2007-Spring), pp. 24092413, Apr. 2007.

[12] H. Li, "Differential space-time-frequency modulation over frequencyselective fading channels," IEEE Commun. Letter, vol. 7, no. 8, pp 349-351, Aug. 2003

[13] J. Wang and K. Yao, "Differential unitary space-time-frequency coding for MIMO OFDM systems," Proc. IEEE 36-th Asilomar Conference on Signals, Systems and Computers, vol. 2, pp. 1867-1871, Nov. 2002.

[14] Q. Ma, C. Tepedelenlioglu, and Z. Liu, "Differential spacetimefrequency coded OFDM with maximum multipath diversity," IEEE Trans. Wireless Commun., vol. 4, no. 5, pp. 2232-2243, Sept. 2005.

[15] S. N. Diggavi, N. Al-Dhahir, A. Stamoulis, and A. R. Calderbank, "Differential space-time coding for frequency-selective channels," IEEE Commun. Letter, vol. 6, no. 6, pp. 253-255, June 2002.

[16] T. Himsoon, W. Su, and K. J. R. Liu, "Single-block differential transmit scheme for broadband wireless mimo-ofdm systems," IEEE Trans. Signal Process., vol. 54, no. 9, pp. 3305-3314, Sept. 2006.

[17] Z. Liu and G. B. Giannakis, "Block differentially encoded OFDM with maximum multipath diversity," IEEE Trans. Wireless Commun., vol. 2, no. 3, pp. 420-423, May 2003

[18] A. Batra, J. Balakrishnan, A. Dabak, and many others, "Multi-band OFDM physical layer proposal for IEEE 802.15 task group 3a," IEEE P802.15-04/0493rl, Sept. 2004.

[19] J. Foerster and Intel R\&D, "Channel modelling sub-committee report final," IEEE P802.15 Working Group for Wireless Personal Area Networks (WPANs), IEEE P802.15-02/490r1-SG3a, Oct. 2005.

[20] R. B. Marks et al., "IEEE tandard for local and metropolitan area networks. Part 16: Air interface for fixed and mobile broadband wireless access systems," IEEE 802.16e-2005, Dec. 2005. 